\title{
COMPANIONS TO HIGH REDSHIFT QUASARS
}

\author{
Esther M. Hu \\ Institute for Astronomy \\ University of Hawaii \\ 2680 Woodlawn Drive \\ Honolulu, HI 96822 USA
}

\begin{abstract}
Lyman $\alpha$ companions to high-redshift quasars appear associated with radio activity in the quasar. Of the five known companion systems seen at typical $\mathrm{L} \alpha$ fluxes of a few $\times 10^{-16}$ ergs $\mathrm{cm}^{-2} \mathrm{sec}^{-1}$, all are associated with quasars which are radio sources, and lie within a few arcseconds of the quasar. We summarize results of searches for the companions, and report on subsequent investigations into the spectral energy distribution and radio studies of these objects. Multi-color imaging from $3400 \AA$ to $2.2 \mu$ shows the companion to PKS $1614+051$ possesses a blue SED similar to an irregular at that redshift. VLA observations show doublelobed structure around Q1033+137 with depolarization of the counterjet lobe.
\end{abstract}

\section{INTRODUCTION}

In searching for high-redshift galaxies it is natural to consider the vicinity of quasars, which form the bulk of the known high- $z$ population. The quasar might be used either as a 'marker' designating a region of red-shift space in the correlated distribution of galaxies or as a 'lighthouse' providing extra illumination for nearby gas and galaxies by processes such as photoionization, dust-scattering of continuum light, or even by inducing star formation. Despite numerous surveys for emission-line companions to distant quasars (Djorgovski et al. 1985; Hu and Cowie 1987; Djorgovski 1988; Kjaergaard et al. 1988) no L $\alpha$ companions were turned up in searches of $\sim 60$ $z>2.5$ quasar systems after the initial successful discovery of a companion to PKS $1614+051$ at $z \sim 3.2$ (Djorgovski et al. 1985). However, most of these candidates were identified by optical selection criteria, and in a recent study where Hu et al. (1991) surveyed roughly equal numbers of radio-loud and optically selected quasars at $z \gtrsim 3$ to homogeneous limits, three new $L \alpha$ companion systems were discovered among the 10 radio-loud quasar systems, while again no companions were found associated with the optically chosen quasars. An additional L $\alpha$ companion emission system to Q1548+096, found serendipitously by Steidel et al. 1990 also shows radio emission from the quasar. The close proximity $\left(<6^{\prime \prime}\right)$ of all these systems to the quasar and the marked association of $\mathrm{L} \alpha$ companions with radio quasars strongly suggests that these objects are linked to the radio quasar phenomenon. 


\section{PROPERTIES OF THE L $\alpha$ COMPANIONS}

Further studies of the nature of the $\mathrm{L} \alpha$ companions have investigated the spectral energy distributions (SEDs) and radio properties of these objects. Two outstanding questions are the nature of galaxies which may underlie the emission systems and comparison of properties with related classes of objects such at the emission systems around low- $z$ quasars and the high- $z$ radio galaxies of similar redshift. SEDs for L $\alpha$ companion systems show some blue objects, with the most complete data for the PKS 1614+051 companion. Multi-color imaging from $3400 \AA$ to $K$-band shows this to have an SED consistent with a red-shifted irregular - substantially bluer than SEDs of known high- $z$ radio galaxies shown in Chambers and Charlot (1990). One recently found $z \sim 2.4$ radio galaxy, Herc 202, may have similar colors (Windhorst 1989).

Emission-line fluxes, separations, and morphologies are similar to those of the low- $z$ quasar systems, which are also found predominantly around radio quasars. These systems might have arisen in tidal interactions of galaxies with the quasar. Alternatively, companions may have been produced in cooling flows. Although none of the $L \alpha$ companion systems are themselves detected radio sources, observations of the Q1033+137 system (Hu, Taylor, and Perley, in preparation) show this quasar to be a Fanaroff-Riley II source, with double-lobed structure roughly aligned along the direction of $L \alpha$ extension, a small nuclear jet, and depolarization of the counter-jet lobe as has been seen for many low- $z$ FR II's. This may be due to a surrounding medium of hot ionized gas of high density, sufficient to produce a cooling flow.

\section{REFERENCES}

Chambers, K. C., and Charlot, 1990, S. Ap. J. (Letters), 348, L1.

Djorgovski, S. 1988, in Towards Understanding Galaxies at Large Redshifts, ed.

R. G. Kron and A. Renzini, (Dordrecht: Kluwer), 259.

Djorgovski, S., Spinrad, H., McCarthy, P., and Strauss, M. A. 1985, Ap. J. (Letters), 299, L1.

Djorgovski, S., Strauss, M. A., Perley, R. A., Spinrad, H., and McCarthy, P. 1987, A. J., 93, 1318.

Hu, E. M., and Cowie, L. L. 1987, Ap. J. (Letters), 317, L7.

Hu, E. M., Songaila, A., Cowie, L. L., and Stockton, A. 1991, Ap. J., 368, 28.

Kjaergaard, P., Möller, P., Jensen, P., and Jörgensen, I. 1989, in IAU Symposium

No. 134, ed. D. E. Osterbrock and J. S. Miller, (Dordrecht: Kluwer), 53.

Windhorst, R. A. 1989, in The Evolution of the Universe of Galaxies, ed. R. G.

Kron, Astr. Soc. Pac. Conf. Ser., 10, 389. 